\title{
Demographic correlates of oral hygiene among stroke survivors undergoing rehabilitation
}

SADJ August 2019, Vol. 74 No. 7 p364 - p369

UI Lawal', R Ibrahim ${ }^{2}$, KJ Ramphoma ${ }^{3}$

\section{ABSTRACT}

\section{Introduction}

Oral-related health does not primarily attract attention among healthcare providers during planning and processing of rehabilitation after stroke.

\section{Aim}

To investigate demographic correlates of oral-hygiene among stroke survivors undergoing rehabilitation.

\section{Methods}

A cross-sectional study was conducted to determine the socio-demographic correlates of oral hygiene in stroke survivors receiving rehabilitation at the National Hospital Abuja (NHA), Nigeria. Sixty stroke survivors participated in this study. Sociodemographic factors of age, sex, education, socioeconomic status, type of stroke, duration of stroke and arm dominance were documented. Oral-hygiene was assessed using the Simplified Oral-Hygiene Index (OHI-S) and the Oral Health Impact Profile 14 (OHIP-14). Descriptive and correlation statistics were used to analyse the data collected.

\section{Author affiliations:}

1. Isa U. Lawal: BSc. (Physiotherapy), MSc.(Ed), PhD., Department of Physiotherapy, Faculty of Allied Health Sciences, College of Health Sciences, Bayero University, Kano, Nigeria. ORCID Number: 0000-0002-2790-5403

2. Rabiu Ibrahim: BSc. (Physiotherapy), MSc., National Assembly Clinic Abuja, Department of Medical Services, Physiotherapy Unit, Abuja, Nigeria.

ORCID Number: 0000-0002-3560-0159

3. Khabiso J. Ramphoma: $B C h D, M C h D$., Department of Community Dentistry, School of Oral Health Sciences, Sefako Makgatho Health Sciences University, Pretoria, South Africa.

ORCID Number: 0000-0001-9975-2912

Corresponding author: Khabiso J. Ramphoma

School of Oral Health Sciences, Sefako Makgatho Health Sciences University, Pretoria, South Africa.

Email: khabiso.ramphoma@smu.ac.za

Author contributions:

$\mathrm{RI}$ and IUL developed and conceived of the study title. IUL and RI were responsible for the study design. All authors (IUL, RI and KJR) were involved in determining and developing study materials and methods. All authors contributed in determining the choice of assessment tools included in the study. RI coordinated the data collection. All authors were involved in drafting of the study manuscript. All authors helped in editing the final submitted manuscript. All authors have read and accepted the manuscript.

1. Isa U. Lawal (IUL): Principal investigator - $40 \%$

2. Rabiu Ibrahim (RI): Research fellow $-36 \%$

3. Khabiso J. Ramphoma (KJR): Critical advisor - $24 \%$

\author{
ACRONYMS \\ ADL: $\quad$ Activities of Daily Living \\ AKTH: Aminu Kano Teaching Hospital \\ Cl: $\quad$ Calculus Index \\ DI: $\quad$ Debris Index \\ NHA: National Hospital Abuja \\ OHIP-14: Oral Health Impact Profile 14 \\ OHI-S: Simplified Oral-Hygiene Index
}

\section{Results}

Results showed that more than half of the participants have had either poor or a fair level of oral-hygiene (23\% and $30 \%$ respectively). Oral hygiene correlated significantly positive with age $(r=0.49)$ but significantly negative with level of education $(r=-0.67)$, socioeconomic status $(r=-0.61)$ and place of residence $(r=-0.55)$.

\section{Conclusion}

Oral hygiene is compromised in stroke survivors undergoing rehabilitation and is largely influenced by old age, poor socioeconomic status, low level of education and poor residential status.

\section{INTRODUCTION}

Stroke has a significant impact on both the physical and psychosocial well-being of survivors. ${ }^{1}$ Traditionally, assessment of the outcome of stroke rehabilitation have been based on indicators of physical function.

Such measures may under-rate the all-inclusive impact of stroke as the focus is only on the physical function, while other health concerns that may arise as a result of the physical impairment are overshadowed.

Individuals who suffered stroke present with different forms of limitations, affecting both the basic and instrumental aspects of Activities of Daily living (ADL), ranging from personal grooming to participation within the community.

These limitations by extension affect specific and general hygiene. One specific aspect of personal hygiene that has received minimal attention, particularly in stroke patients undergoing rehabilitation, is the status of oral hygiene. ${ }^{2}$ 
Individuals who suffered stroke may have difficulty in independently completing the requisite oral health care tasks because of hemiparesis, hemiplegia, poor grip, ineffective use of the non-dominant hand when this has been spared, lack of coordination, or cognitive deficits. $^{3}$

Consequently and in particular when stroke is accompanied by facial palsy, food can become trapped in the buccal cavity without the patient being aware of the accumulation. Additionally, if optimal brushing is difficult, dental caries and periodontal disease can occur as a result of the increased bacterial load. Moreover, as the saliva becomes populated by high rates of bacteria, these may be aspirated through dysphagia or other conditions, and pneumonia or systemic infection can result. ${ }^{2}$

The literature has evidence that poor oral hygiene occurs among stroke survivors, and that it has been identified as a cause of aspiration pneumonia, systemic infection, endocarditis and even death in patients who have suffered stroke..$^{2,3}$

Notably, the majority of these studies were conducted in the developed parts of the world. There is only limited data from the developing nations on this topic, resulting in a poor evaluation of this crucial aspect of stroke care.

This study investigated the relationship between oral hygiene status and some socio-demographic and clinical characteristics (laterality, duration and type of stroke) in a population of stroke survivors from two Physiotherapy outpatient departments in Nigeria.

\section{METHODS}

A cross-sectional survey was conducted on oral hygiene among individuals who had suffered a stroke, and who were attending the Physiotherapy out-patient department of Aminu Kano Teaching Hospital (AKTH), Kano, Nigeria. Participants were sampled through the convenience sampling technique.

Patients who met the inclusion criteria of being diagnosed with a first ever unilateral stroke according to the definition of stroke according to the World Health Organization 4 (and screened by a physician), were $\geq 18$ years of age; with stroke onset $\geq 14$ days; having a score of $\geq 19$ points on the Mini Mental State Examination, ${ }^{5}$ were included as participants in the study.

Excluded patients were those still smoking at the time the study was conducted or those who had quit smoking $\leq 3$ months ago and/or had lost at least half of their teeth.

\section{Procedure}

A written informed consent document was developed, and it was ensured that all participants read and understood the procedures, and were allowed to ask any possible question(s) before signing consent. Only then did data collection proceed.
The demographic features of each participant were recorded. Assessment of participants for oral hygiene related measures was conducted as detailed below:

Two dental therapists used the Simplified Oral Hygiene Index (OHI-S) to measure oral hygiene status of each patient. The Index is composed of a combination of the Debris Index (DI) and the Calculus Index (Cl).

Each of these indices is based on numerical determinations representing the amount of debris or calculus found on the buccal and lingual surfaces of the preselected tooth.

The maxillary and mandibular arches are examined in three segments; each being examined for debris and/or calculus. Six surfaces were selected from the four posterior and two anterior teeth for examination for $\mathrm{OHI}-\mathrm{S}$. In the posterior portion of the dentition, usually the first molar but sometimes the second or third molar was examined.

The buccal surfaces of the selected upper molars and the lingual surfaces of the selected lower molars were inspected. In the anterior portion of the mouth, the labial surfaces of the upper right and the lower left central incisors were scored.

For each individual, the debris scores were totaled and divided by the number of surfaces scored. At least two of the six possible surfaces had to be scored and examined before an individual score were calculated. The same methods were used to obtain the calculus score.

The $\mathrm{DI}$ and the $\mathrm{Cl}$ were combined to obtain the $\mathrm{OHI}-\mathrm{S}$. The $\mathrm{Cl}$ and $\mathrm{DI}$ values may range from 0 to 3 ; the $\mathrm{OHI}-\mathrm{S}$ values from 0 to 6 , with lower scores indicating better oral hygiene. The OHI-S is the gold standard for the assessment of oral hygiene. ${ }^{6}$

The data were initially captured in Microsoft Excel 2007; coded in numeric and were exported to SPSS version 20.0 for analysis, including both descriptive and inferential statistics. Visual inspections of box plots, histograms, and skewedness were used to determine variable normality and homoscedasticity. Participants' socio-demographic and study outcome measures were described using frequencies, percentages, means and standard deviations as the case may be for either categorical or continuous variables.

Pearson's moment correlation coefficient and Spearman's Ranking were used to determine the statistical significance and strength of the relationship between variables. The strength of the relationship between independent and dependent variables was described using the correlation coefficient $(r)$ and was based on Cohen's interpretations. ${ }^{7}$ A p-value $\leq 0.05$ was deemed statistically significant in all calculations.

Ethical clearance for the study was obtained from the Ethics Research Committee of Aminu Kano Teaching Hospital, Kano with ethical reference number; NHREC/ 21/08/2008/AKTH/EC/1615 


\section{RESULTS}

Demographic features

Sixty stroke survivors participated in this study.

The sample consisted of 32 (53.3\%) males and 28 $(46.7 \%)$ females. The mean age of the participants was $55.7 \pm 12.9$ years (range $=22$ to 82 years).

Approximately $66.7 \%$ of the subjects $(n=40)$ had suffered a stroke more than six months prior to the study, while the remaining 23.3\% $(n=14)$ and $10 \%(n=6)$ had respectively undergone stroke onset between three to six months and less than three months prior to the study.

Most participants $(66.7 \%, n=40)$ presented with right sided hemiparesis. The majority of the participants had suffered ischaemic stroke (53.5\%). Thirty-five stroke survivors (58.3\%) had hemiparesis affecting the dominant upper extremity and 25 (41.7\%) showed effects on the non-dominant side (Table 1).

\begin{tabular}{|c|c|c|c|}
\hline Variables $(n=60)$ & \multicolumn{2}{|c|}{ n (\%) } & OHI-S \\
\hline \multicolumn{4}{|l|}{ Gender } \\
\hline Male & 32 & (53.3) & $2.42 \pm 1.34$ \\
\hline Female & 28 & $(46.7)$ & $2.22 \pm 0.91$ \\
\hline \multicolumn{4}{|l|}{ Level of education } \\
\hline None & 14 & $(23.3)$ & $3.82 \pm 1.32$ \\
\hline Primary & 8 & $(13.3)$ & $2.04 \pm 0.70$ \\
\hline Secondary & 10 & $(16.7)$ & $2.22 \pm 0.54$ \\
\hline Tertiary & 28 & $(46.7)$ & $1.71 \pm 0.51$ \\
\hline \multicolumn{4}{|c|}{ Socio-economic status } \\
\hline Lower & 18 & $(30.0)$ & $3.43 \pm 1.26$ \\
\hline Middle & 36 & $(60.0)$ & $1.92 \pm 0.73$ \\
\hline Higher & 6 & $(10.0)$ & $1.47 \pm 0.22$ \\
\hline \multicolumn{4}{|l|}{ Place of residence } \\
\hline Urban & 41 & (68.3) & $1.91 \pm 0.79$ \\
\hline Rural & 19 & (31.7) & $3.23 \pm 1.31$ \\
\hline \multicolumn{4}{|l|}{ Type of stroke } \\
\hline Hemorrhagic & 28 & $(46.7)$ & $2.47 \pm 1.26$ \\
\hline Ischemic & 32 & $(53.5)$ & $2.20 \pm 1.05$ \\
\hline \multicolumn{4}{|l|}{ Duration of stroke } \\
\hline$<3$ months & 6 & (10.0) & $1.75 \pm 1.13$ \\
\hline 3 to 6 months & 14 & (23.3) & $2.17 \pm 0.89$ \\
\hline$>6$ months & 40 & $(66.7)$ & $2.47 \pm 1.23$ \\
\hline \multicolumn{4}{|c|}{ Side affected/arm dominancy } \\
\hline Dominant side & 35 & $(58.3)$ & $1.75 \pm 1.13$ \\
\hline Non-dominant side & 25 & $(41.7)$ & $2.17 \pm 0.89$ \\
\hline
\end{tabular}

\section{Oral hygiene and socio-demographic} characteristics

Study findings showed a significant correlation between age and oral hygiene $(r=0.492, p<0.001)$.

Further-more, significant relationships were observed between the level of education attained by stroke survivors $(r=-0.672, p<0.001)$; level of income $(r=-0.608$, $p<0.001)$; place of residence $(r=0.550, p<0.001)$ and oral hygiene (Table 2).

\begin{tabular}{|c|c|c|}
\hline Variable & OHI-S & p-value \\
\hline Age & $0.492^{\star \star \star}$ & $<0.001$ \\
\hline Gender & -0.13 & 0.924 \\
\hline Level of education & $-0.672^{\star \star \star}$ & $<0.001$ \\
\hline Level of income & $-0.608^{\star \star \star}$ & $<0.001$ \\
\hline Place of residence & $0.550^{\star \star *}$ & $<0.001$ \\
\hline Duration of stroke & 0.200 & 0.120 \\
\hline Type of stroke & -0.115 & 0.381 \\
\hline Side affected/arm dominancy & -0.163 & 0.215 \\
\hline
\end{tabular}

\section{DISCUSSION}

This study investigated the demographic and clinical correlates of oral hygiene post stroke. The findings indicate that oral hygiene has significant correlations with age, level of educational attainment, socioeconomic status, community of residence and laterality.

On the other hand, dominance, gender, onset and type of stroke were shown to have no significant association with oral hygiene among individuals who have suffered stroke.

The majority of the participants in this study were male, which is in line with a previous study carried out in Kano, in Northwestern Nigeria, in which the the number of male stroke survivors was almost twice the number of female participants. ${ }^{8}$ Generally, stroke is more prevalent in men than women in most age groups except for very old age, greater than 80 years..$^{9,10,11}$ This epidemiologically established gender difference in the distribution of stroke is reflected in this study.

The mean age and age range $(55.7 \pm 12.9)$ of the participants is also similar to those reported in a previous study $(55.0 \pm 16.5) .{ }^{8}$ Stroke is mainly a disease of the elderly and the majority of those affected were in their fifth decade of life or more. ${ }^{12}$ Additionally, $58 \%$ of participants in this study presented with the dominant side affected, specifically suggesting left hemispheric lesion.

A previous study reported a higher frequency of left hemispheric ischaemic stroke, at 54\%. ${ }^{13}$ The most common stroke type among participants in this study is ischaemic (54\%) and this is consistent with several epidemiologic studies. ${ }^{8,14-16}$

The findings of the current study indicated that older stroke patients presented with poorer oral hygiene. Old age as a stage in life is associated with declining personal hygiene which has been linked to self-neglect. ${ }^{17}$ Age challenges have been found to influence the ability of stroke survivors to perform self-care. ${ }^{18}$

Even in the absence of stroke, approximately, $40 \%$ of individuals aged 60 years and above need some assistance to perform at least one instrumental activity of daily living. ${ }^{19}$

This is expected to worsen when stroke sets in and, as social experience confirms; it is very common that the older one gets, the lesser the concern with 
personal hygiene, especially oral hygiene. Hence, in the presence of stroke which can disable its survivors, oral hygiene tends to be forgotten or neglected completely.

Therefore, the finding of poorer oral health status among older stroke patients is not unexpected, calling for further attention to the oral hygiene of especially elderly stroke survivors by the health professionals and family caregivers. This finding is consistent with another study where older stroke survivors were reported to show a poor state of oral health. ${ }^{20}$

There was no significant gender relationship reflected in the oral hygiene of stroke survivors, contrary to findings among healthy individuals where gender was found to be a major determinant of oral hygiene status, with females having the better record. ${ }^{21}$ However, a previous study demonstrated that gender was not associated with utilization of oral health services (such as dental visitation) among healthy Nigerian adults. ${ }^{22}$

It is believed that the better oral hygiene reported among healthy women is specifically attributed to enhanced oral hygiene-seeking behaviour exhibited by women as compared with men. ${ }^{23}$ Our results showed that this oral hygiene-seeking behaviour is not differentiated between genders and therefore, gender does not influence the oral hygiene status among stroke survivors. This empasises that equal attention in oral health should be given to both male and female stroke survivors undergoing rehabilitation.

The level of educational attainment was found to be significantly related to the oral hygiene status of stroke survivors; the higher the level of education (tertiary) the better the oral hygiene status. This finding is similar to the finding of another study which alluded to the fact that lack of tertiary education was significantly associated with poor oral health. ${ }^{24}$ There is a correlation between educational attainment, socioeconomic status, urbanization and oral health status. ${ }^{25}$ Having acquired higher education may have the advantage of exposing an individual to the knowledge and importance of oral health, resulting in the individual exercising greater attention to oral hygiene. In contrast, when knowledge is lacking, not much attention will be given to the oral health until such time when a discomforting condition is developed.

Level of income is a major determinant of economic status; the study results showed that about $37 \%$ of oral hygiene status is "explained" by the economic status of the individual. Findings indicated that participants belonging to the lower economic class presented with significantly poorer oral hygiene status compared with those belonging to the middle and upper economic classes. However, a previous study reported that the impact of education on oral health is much stronger than the financial capability. ${ }^{26}$

Nevertheless, in most developing countries such as Nigeria, financial capacities at times do have a greater impact on the social wellbeing of an individual. The more financially buoyant an individual, the more ready the access to social/health services. Stroke survivors of the low income class will be left overwhelmed by the costs of treatments and rehabilitation services for their condition and therefore, may have challenges in attending to their oral hygiene or oral health.

This can happen to even those stroke survivors who have attained a sufficient level of education but are of low income class. They may be left with the only option of depending on local means of oral hygiene care practices such as the use of chewing sticks, ashes or charcoal powder which may be inadequate in maintaining sufficient oral hygiene..$^{27,28}$

Place or community of residence has an impact on the oral hygiene of stroke survivors, those who reported living in urban communities showed significantly better oral hygiene status compared with those living in the rural communities. This finding is in agreement with another study that showed that urban people were more likely to have their teeth cleaned compared with rural people. ${ }^{29}$

This could be explained by the fact that traditionally, in developing countries such as Nigeria, and particularly in the Northern part, individuals who belong to the high socio-economic class and those who attained a higher level of education tend to live in urban areas, even if they originated from rural communities. The existence of social amenities, potentialities and opportunities attracted them to the urban areas, consequently leaving the rural communities populated with a lower socio-economic class.

Most of the rural communities are lacking basic social amenities such as oral health care facilities and items used in achieving good oral hygiene like tooth paste and tooth brushes. Stroke survivors who belong to such rural communities may therefore not have adequate access to such items and facilities. Perhaps only those few educated individuals may have to depend on the local means of oral health care. As reiterated earlier, financial capacity and knowledge play roles in one's ability to keep a good oral hygiene. This calls for more attention to be given to oral health during the assessment and rehabilitation of individuals who suffered stroke, particularly those living in rural communities.

It is expected that those stroke survivors with the dominant arm affected might not be able to carry out optimal brushing or mouth cleaning and as such may have poorer oral hygiene. In contrast, this study finding showed that the affected side has no relationship with oral hygiene maintenance in stroke survivors. This finding is in agreement with a previous study which concluded that paralysis of the dominant hand had no added effect on physical functioning in stroke survivors. ${ }^{30}$ Moreover, our study findings indicated that even at three months after onset of stroke, involvement of the dominant arm may have no impact on ADL such as oral hygiene care practices.

However, it is worth noting that some of these stroke survivors are indeed not able to take care of their oral hygiene, and might have to depend on their caregivers for the task. If the latter is dedicated and efficient, the 
side affected might not be a hindrance to good oral hygiene, and perhaps this explains the finding of this study; that the side affected has no impact on the oral hygiene of stroke survivors.

The type of stroke (ischaemic versus hemorrhagic) was also demonstrated to have no impact on the oral hygiene of stroke survivors. Both ischemic and hemorrhagic stroke survivors were shown on average to have fair oral hygiene status. This finding is not unexpected, as the type of stroke may not necessarily denote the severity of manifestation, especially at a chronic stage. The area and the extent of the involvement in the brain will rather determine the severity of the manifestation. Even though a previous study reported evidence of better functional prognosis in stroke survivors with hemorrhagic stroke, ${ }^{31}$ our findings indicated that the type of stroke was not related to oral hygiene.

Duration (time since the occurrence of stroke) amongst stroke survivors was shown to have no relationship with oral hygiene status. This finding is in the direction of a previous investigation, where the authors demonstrated no significant differences in the Calculus-Plaque index scores between participants having different durations since stroke. ${ }^{13}$ These findings are not unexpected as there are stronger factors that determine the oral hygiene status of stroke survivors; such as socioeconomic status, level of education, age, family and whether the patient is an acute, sub-acute or chronic stroke survivor.

The oral hygiene status will depend on these factors rather than the duration of time since the occurrence of the stroke. Also, family caregivers, when present and active, can mask the effect of time on the general hygiene (including oral hygiene) of the stroke survivor. Therefore the implication of these findings is that attention should be given on the oral hygiene of all stroke survivors from day one, irrespective of the duration of the stroke.

\section{CONCLUSION}

A number of stroke survivors undergoing rehabilitation do present with poor oral hygiene. Several sociodemographic factors (level of education, level of income, age and community of residence) recorded a significant relationship with the oral hygiene status post-stroke. Therefore, those stroke survivors with poor formal education, low level of income, old age and/or living in the rural areas may require extra attention specifically on oral hygiene and general hygiene during rehabilitation planning and subsequent care.

\section{References}

1. Draper P, Min T, Phil B. The impact of stroke on the wellbeing of the patient's spouse. An exploratory study. J Clin Nurs. 2007; 16 (2): 264-71.

2. Kwok C, Mclntyre A, Janzen S, Mays R, Teasell, R. Oral care post- stroke: A scoping review. J Oral Rehabil. 2015; 42: $65-74$

3. Hunter RV, Clarkson JE, Fraser HW, MacWalter RS. A preliminary investigation into tooth care, dental attendance and oral health related quality of life in adult stroke survivors in Tayside, Scotland. Gerodontology 2006; 23(3): $140-8$.
4. WHO MONICA Project Investigators. The World Health Organization MONICA Project (Monitoring trends and determinants in cardiovascular disease). J Clin Epidemiol. 1988; 41: 105-14.

5. Mungas D. In-office mental status testing: a practical guide. Geriatrics 1991; 46: (7), 54-8, 63, 66.

6. Cascaes AM, Peres KG, Peres MA, et al. Validity of 5-year old children's oral hygiene pattern referred by mothers. Rev Saude Publica. 2011; 45(4): 668-75.

7. Cohen J. Statistical Power Analysis for the Behavioral Sciences, 2nd ed. Hillsdale, NJ: Lawrence Erlbaum Associates, 1988

8. Owolabi LF, Nagoda M. Stroke in developing countries: experience at Kano, Northwestern Nigeria. Sudan J Med Sci. 2012; 7 (1): 9-14.

9. Bogousslavsky J, Van Melle G, Regli F. The Lausanne Stroke Registry: analysis of 1,000 consecutive patients with first stroke. Stroke 1988; 19(9): 1083-92.

10. Reeves M J, Bushnell CD, Howad G et al. Sex differences in stroke: epidemiology, clinical presentation, medical care and outcomes. The Lancet Neurol. 2008; 7(10): 925-6.

11. Foerch C, Ghandehari K, Xu G, Kaul S. Exploring gender distribution in patients with acute stroke: a multi-national approach. J Res Med Sci. 2013; 18(1): 10-6.

12. Engstad T, Viitanen M, Ellekjær H. Epidemiology of stroke in the elderly in the Nordic countries. Incidence, survival, prevalence and risk factors. Norsk Epedemiologi 2012; 22(2). https://doi.org/ 10.5324/nje.v22i2.1557.

13. Hedna VS, Bodhit AN, Ansari S, Falchook AD, Stead L, Heilman KM, and Waters MF. Hemispheric differences in ischemic stroke: is left-hemisphere stroke more common? J Clin Neurol. 2013; 9(2): 97-102. doi: 10.3988/ jcn.2013.9.2.97.

14. Appleton JP, Sprigg N, Bath PM. Blood pressure management in acute stroke. Stroke and Vascular Neurology. 2016; 1(2):72-82.

15. Amarenco P, Bogousslavsky J, Caplan LR, Donnan GA, Hennerici MG. Classification of stroke subtypes. Cerebrovascular Diseases 2009; 27(5): 493-501.

16. Warlow CP. Epidemiology of stroke. The Lancet. 1998; 1;352:S1-4

17. Yunus GY, Itagi AH. Oral health status in cerebrovascular accident survivors : A descriptive cross-sectional study. Ind J Sci Res Tech. 2014; 2(1): 61-8.

18. British Society of Gerontology. Guidelines for the Oral Healthcare of Stroke Survivors 2010, [cited 2018 Oct 19]. Available from: https//WWW.gerontology.com.

19. da Silva FC, da Silva FT, Mesquita-Ferrari RA, Fernandes PS, Bussadori SK. Correlation between upper limb function and oral health impact in stroke survivors. J Physical Ther Sci. 2015; 27(7): 2065-8.

20. Touza C, Prado C. Detecting self-neglect: a comparative study of indicators and risk factors in a Spanish population. Gerontology and Geriatric Medicine 2019; 5: 1-10. DOI: 10.1177/2333721418823605.

21. da Silva SR, Castellanos Fernandes RA. Self-perception of oral health status by the elderly. Rev Saude Publica. 2001; 35(4): 349-85

22. Olabisi A, Udo U, Adeniyi A, Bashiru B, Ehimen U, Gbenga O. Prevalence of dental caries and oral hygiene status of a screened population in Port Harcourt, Rivers State. Nig J Int Soc Prev and Comm Dent. 2015; 5(1): 59. https://doi. org/10.4103/2231-0762.151979.

23. Olusile AO, Adeniyi AA, Orebanjo O. Self-rated oral health status, oral health service utilization, and oral hygiene practices among adult Nigerians. BMC Oral Health 2014; 14(1). https://doi.org/10.1186/1472-6831-14-140.

24. Sogi G, Bhaskar DJ. Dental carries and oral hygiene status of 13-14 year old school children of Davangere. J Ind Soc Pedodon and Prev Dent. 2001; 19: 113-7.

25. Lam OL, McMillan AS, Li LS, McGrath C. Predictors of oral health-related quality of life in patients following stroke. Journal of Rehabilitation Medicine 2014; 46: 520-526. 
26. Miao J, Wu X. Urbanization, socioeconomic status and health disparity in China. Health and Place 2016; 42: 87-95.

27. Asgari F, Majidi A, Koohpayehzadeh J, Etemad K, Rafei A. Oral hygiene status in a general population of Iran, 2011: A key lifestyle marker in relation to common risk factors of non-communicable diseases. Int $\mathrm{J}$ Health Poli and Manag. 2015; 4(6): 343-52.

28. Fukada H, Saito T, Kihara E, Ogada C, Wagaiyu EG, Hayashi Y. Oral hygiene status of chewing stick users in a rural Kenyan community. Oral Health Den Manag. 2016; 15(2)

29. Brooks JK, Bashirelahi N, Reynolds MA. Charcoal and charcoal-based dentifrices: A literature review. J Am Den Assoc. 2017; 148 (9): 661-70.

30. Nam HU, Huh JS, Yoo JN, et al. Effect of dominant hand paralysis on quality of life in patients with subacute stroke. Ann Rehabil Med. 2014; 38 (4): 450-7.

31. Paolucci S, Antonucci G, Grasso MG, et al. Functional outcome of ischemic and hemorrhagic stroke patients after in-patient rehabilitation: a matched comparison. Stroke 2003; 34(12): 2861-5.

\section{Do the CPD questionnaire on page 405}

The Continuous Professional Development (CPD) section provides for twenty general questions and five ethics questions. The section provides members with a valuable source of CPD points whilst also achieving the objective of CPD, to assure continuing education. The importance of continuing professional development should not be underestimated, it is a career-long obligation for practicing professionals.

\section{Online CPD in 6 Easy Steps}

\section{Go to the SADA website www.sada.co.za.}

2 Log into the 'member only' section with your unique SADA username and password.

3 Select the CPD navigation tab.

4 Select the questionnaire that you wish to complete.

5 Enter your multiple choice answers. Please note that you have two attempts to obtain at least $70 \%$.

6 View and print your CPD certificate. 